\title{
Synthesis of Nanostructures using Electron Beam Induced Deposition
}

\author{
P. Kruit ${ }^{*}$ W.F. van Dorp ${ }^{*}$, C.W. Hagen ${ }^{*}$, P.A. Crozier ${ }^{* *}$ \\ * Delft University of Technology, Fac. Appl. Sci, Lorentzweg 1, 2628CJ Delft, The Netherlands \\ Arizona State University, School of Materials, Tempe, AZ 85287-1704
}

Nanostructures can be fabricated in-situ in the electron microscope by Electron Beam Induced Deposition (EBID. A precursor gas is admitted in the vacuum of the microscope, and adsorbed precursor molecules are dissociated by both the primary and the secondary electrons, leaving nonvolatile parts behind on the sample (Figure 1). Different precursor gases allow to deposit Pt, W, Au, etc. The deposits usually contain a large percentage of carbon. The old-fashioned carbon contamination deposits in electron microscopes is also a form of EBID. To obtain the smallest possible deposits, we studied the electron-sample interaction and carefully tuned the process parameters. We performed EBID experiments in the environmental Scanning Transmission Electron Microscope (STEM) of ASU at a beam energy of $200 \mathrm{keV}$, a $0.3 \mathrm{~nm}$ probe and $\mathrm{W}(\mathrm{CO})_{6}$ as a precursor gas. Typical precursor gas pressures during the deposition are $10^{-3}$ Torr, and to reduce contamination from the microscope the substrates are held at an elevated temperature of 100-150 C. We deposited arrays of nanometer sized dots on thin membranes of SiN, amorphous carbon or graphite[1]. The imaging and monitoring of the growth were performed with the annular dark field (ADF) signal. The smallest deposits we have made so far have an average volume of $0.4 \mathrm{~nm}^{3}$.

Structures as small as a few nanometers can be deposited with good dimensional control (figure 2). One of the conditions for obtaining these small structures is that they should not be too tall (aspect ratios below about 2) in order to prevent broadening from electron scattering in the structure itself. When the structure size approaches $1.0 \mathrm{~nm}$, several kinds of non-linearities occur. To understand these findings we studied the nucleation of the deposits (Figure 3). For instance, when an array of deposits is created with a fixed dwell time, the amount of mass varies from deposit to deposit. This distribution of masses becomes wider as the dwell time decreases and the deposits contain less material [2]. We attribute this effect to the intrinsic randomness in the number of molecules that are deposited (shot noise). We have also tried to use the ADF signal to control the deposition by blanking the beam after a preset amount of mass has been deposited [3].

Applications of this technique are pursued in depositing catalysts for nanotube growth and arrays of binding sites for biomolecules. For the moment it seems one of the very few technologies for direct synthesis of arbitrary size structures in the 1-10 nm range in controlled positions.

[1] W.F. van Dorp, B. van Someren, C.W. Hagen, P. Kruit, P.A. Crozier, Nano. Lett. 5 (2005) 1303

[2] W.F. van Dorp, B. van Someren, C.W. Hagen, P. Kruit, P.A. Crozier, J Vac Sci Technol B 24 (2006) 618

[3] W.F. van Dorp, C.W. Hagen, P.A. Crozier, P. Kruit; J Vac Sci Techn B 25 (2007) 2210 


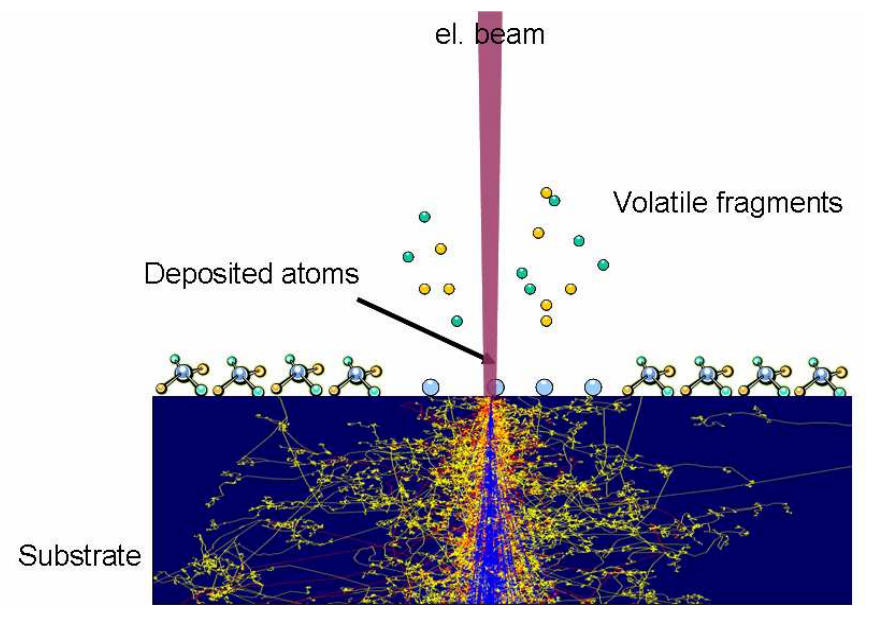

FIG.1: The principle of EBID

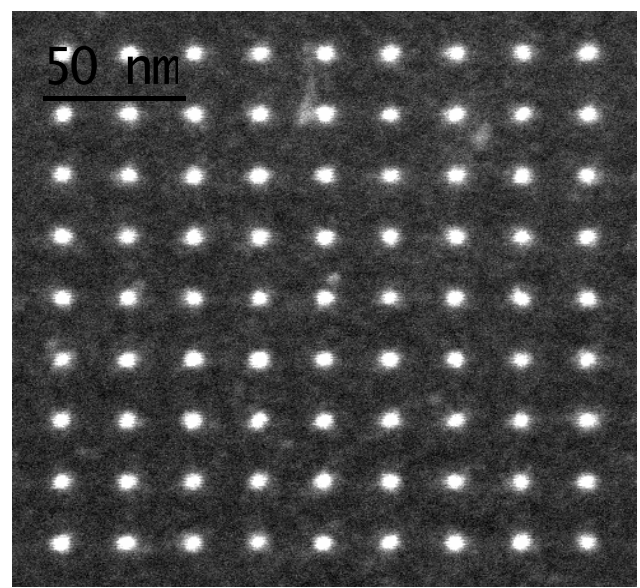

FIG.2: $4.5 \mathrm{~nm}$ diameter $\mathrm{W}$ dots on $\mathrm{C}$ foil at $11 \mathrm{~nm}$ half pitch
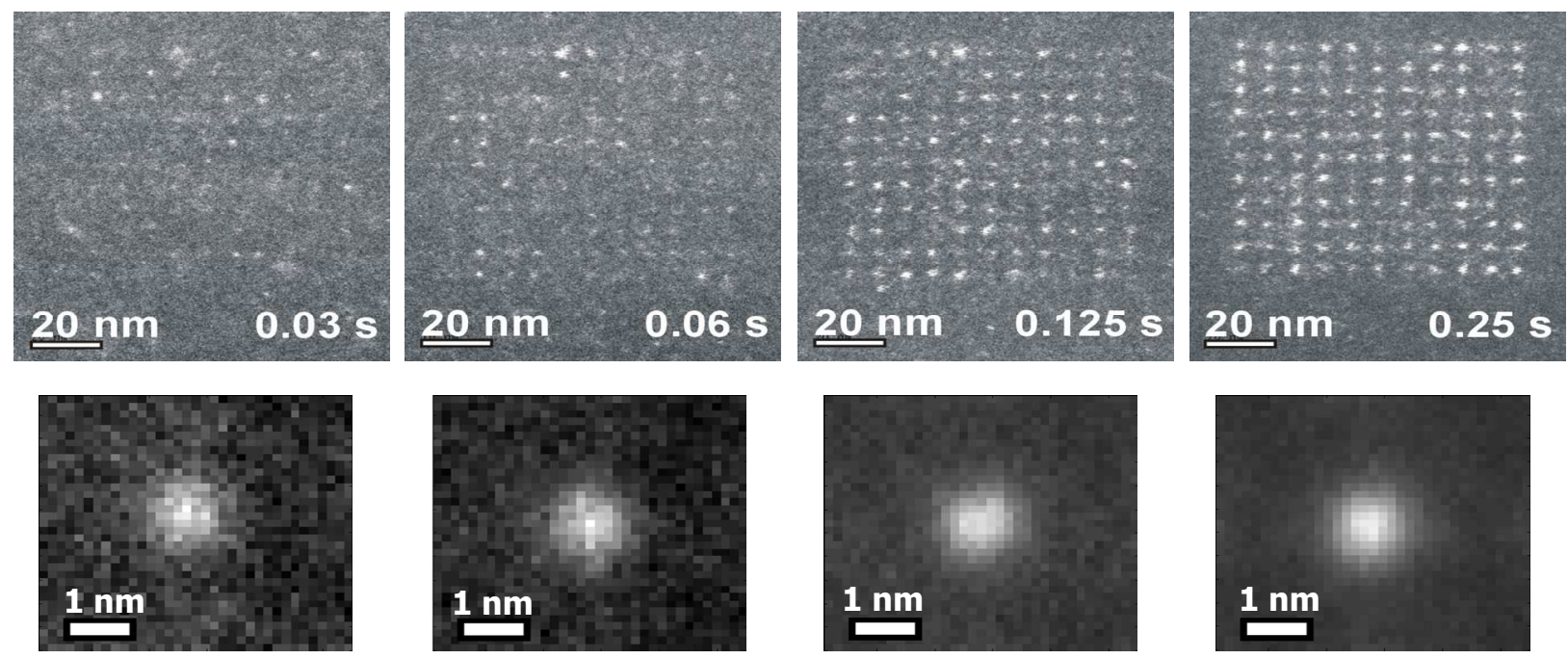

FIG.3: Through-dose series of $1 \mathrm{~nm}$ dots, both showing the resolution capabilities of EBID and the intrinsic limitation by the fluctuations in number of deposited molecules. The bottom row of pictures shows the avarage dotsize. 